RELATO DE CASO

ISSN 1677-5090

(C) 2019 Revista de Ciências Médicas e Biológicas

http://dx.doi.org/10.9771/cmbio.v18i3.34481

\title{
Mediastinite descendente necrosante pós-angina de Ludwig: relato de caso
}

\author{
Descending necrotizing mediastinitis after Ludwig's angina: case report
}

\begin{abstract}
Patrícia Fonseca Guedes Conceição ${ }^{1}$, Pedro Antonio de Jesus Moureira², Patrícia Miranda Leite Ribeiro ${ }^{3^{*}}$
${ }^{1}$ Mestranda em Processos Interativos dos Órgãos e Sistemas, Instituto de Ciências da Saúde, Universidade Federal da Bahia. ${ }^{2}$ Especialista em Atenção Hospitalar, Hospital Universitário Professor Edgard Santos, Universidade Federal

Bahia. ${ }^{3}$ Doutora em Odontologia, Universidade Federal da Bahia.
\end{abstract}

\begin{abstract}
Resumo
Introdução: a mediastinite necrosante descendente é um processo infeccioso grave do mediastino e pode ser originária de uma infecção odontogênica, apresentando; alta taxa de mortalidade. O cirurgião-dentista deve buscar estratégias para prevenir focos infecciosos, diminuindo a incidência de infeç̧ões odontogênicas graves que se disseminem pelos espaços cervicofaciais e torácicos. Objetivo: relatar caso de um paciente com diagnóstico de mediastinite necrosante pós-angina de Ludwig, descrever suas manifestações clínicas e a conduta terapêutica odontológica adotada. Relato de caso: paciente, 31 anos, sexo masculino, foi admitido referindo sintomatologia dolorosa no dente 3.8, que evoluiu para aumento de volume em região cervical, associado à febre e disfagia progressiva. Ao exame físico extraoral, observam-se sinais de disseminação para os espaços cervicofaciais e torácicos. Após exames complementares, o diagnóstico revela a infecção, associada à angina de Ludwig, secundária à pericoronarite. 0 tratamento foi analgesia e antibioticoterapia por via venosa. Após remissão dos sintomas, o paciente recebeu alta hospitalar em bom estado e foi realizada exodontia do dente 3.8. Conclusão: a mediastinite necrosante é uma patologia rara que pode levar a óbito, se não tratada adequadamente. 0 cirurgiãodentista deve estar consciente da possibilidade de disseminação pelos espaços faciais e mediastinais dos processos infecciosos de origem odontogênica e estar apto para tratar corretamente os casos antes, que eles acarretem grande risco à vida dos pacientes. Palavras-chave: Mediastinite. Angina de Ludwig. Tratamento.
\end{abstract}

\begin{abstract}
Introduction: descending necrotizing mediastinitis (DNM) is a severe infectious process of the mediastinum and may originate from an odontogenic infection; having a high mortality rate. The dentist should seek strategies to prevent infections that spread through the cervicofacial and thoracic spaces. Objective: to report a case of a patient with a diagnosis of DNM after Ludwig's angina, to describe its clinical manifestations and the adopted dental treatment. Case report: a thirty-one year old male patient was admitted with painful 3.8 tooth symptoms that evolved to swelling in the cervical regions, associated with fever and progressive dysphagia. Extraoral physical examination shows signs of spread to the cervicofacial and thoracic spaces. After further examination, the diagnosis was DNM associated with Ludwig's angina secondary to pericoronitis. Treatment was intravenous analgesia and antibiotic therapy. After remission of symptoms, the patient was discharged from hospital in good condition and 3.8 tooth extraction. Conclusion: DNM is a rare condition that can lead to death if not treated properly. The dentist should be aware of the possibility of spreading through the facial and mediastinal spaces of infectious processes of odontogenic origin, being able to correctly treat the cases, before they pose a great risk to patients' lives.

Keywords: Mediastinitis. Ludwig's Angina. Treatment
\end{abstract}

\section{INTRODUÇÃO}

As infecções graves que atingem o complexo cervicofacial são caracterizadas pela disseminação do processo infeccioso pelos tecidos adjacentes e espaços faciais da região de cabeça e pescoço. Elas podem ser classificadas, de acordo com sua origem, em infecções odontogênicas ou não odontogênicas. As infecções odontogênicas são polimicrobianas e oriundas de tecidos dentais e periodontais; podem ser sintomáticas ou assintomáticas e exibir tumefações locais ou generalizadas (AZENHA et al., 2012).

Correspondente/Corresponding: *Patrícia Miranda Leite Ribeiro End: - End: Av. Araújo Pinho, 62 Canela, Salvador - BA, CEP: 40301-155 Faculdade de Odontologia - Tel: (71) 99141-7949 - Email: patrícia. leiteribeiro@gmail.com
Pacientes portadores de doenças crônicas, como diabetes mellitus, infecção pelo vírus da imunodeficiência humana (HIV) e linfomas possuem maior probabilidade de ocorrência de infecções graves, por estarem com o sistema imunológico comprometido (LITTLE et al., 2017). Como meio auxiliar de diagnóstico e para guiar a drenagem cirúrgica, devem ser solicitados exames imaginológicos, visando avaliar os espaços faciais envolvidos. A ultrassonografia de tecidos moles pode ser solicitadas, por ser uma modalidade de exame de imagem ideal para diferenciar infecções superficiais de infecções profundas mais severas. A tomografia computadorizada é um importante método auxiliar no diagnóstico, pois evidencia a extensão da lesão e o comprometimento das vias aéreas 
(TUCUNDUVA et al., 2013).

O manejo da via aérea nas infecções cervicofaciais profundas deve ser cuidadoso. Esse tipo de infecção se torna um desafio para o profissional devido à complexidade anatômica da região, da localização profunda que dificulta o diagnóstico e o acesso cirúrgico e também pela facilidade de disseminação da infecção pelos espaços faciais adjacentes, o que leva a uma alta taxa de mortalidade (PEREIRA et al., 2011).

Uma questão importante para o sucesso do tratamento é a escolha correta do antibiótico a ser utilizado. $O$ antibiótico de escolha para as infecções de origem odontogênica é a penicilina ou, em casos de pacientes alérgicos, a clidamicina.

Dependendo da severidade da infecção é comum a utilização de macrolídeos e cefalosporinas. A associação de penicilina com metronidazol é bastante empregada nos casos de infecções severas, com presença de coleção purulenta, pelo fato de o metronidazol combater com eficácia as bactérias anaeróbias. Contudo, a antibioticoterapia sozinha não parece ser eficaz no tratamento de infecções cervicais profundas, sendo necessária a drenagem cirúrgica (SUEHARA et al., 2008).

A infecção odontogênica pode levar a algumas complicações como: angina de Ludwig, celulite orbital, trombose do seio cavernoso, endocardite bacteriana, mediastinite e sepse. Um estudo revelou o tempo médio de 8 dias de internação hospitalar para pacientes diagnosticados com infecções odontogênicas, referindo que a extensão da infecção e a necessidade de incisão extraoral para drenagem são fatores determinantes para o aumento desse período de internação (RASTENIENÈ et al., 2015).

As infecções odontogênicas graves parecem ter um impacto significativo nas despesas hospitalares e muitas vezes os seus custos são subestimados. Avaliações já foram realizadas para definir o ônus financeiro das infecções odontogênicas, em serviços públicos de saúde pelo mundo (AHMAD et al., 2013; CHRISTENSEN et al., 2013, EISLER et al., 2013; JUNDT et al., 2012).

O objetivo do presente estudo é relatar o caso clínico de um paciente acometido por mediastinite descendente necrosante (MDN) pós-angina de Ludwig, diagnosticado e tratado no Complexo Hospitalar Professor Edgard Santos (Complexo-HUPES).

\section{RELATO DE CASO}

Paciente T.S.S, 31 anos, faioderma, sexo masculino, previamente hígido, foi admitido referindo que há 14 dias iniciou quadro dor no dente 3.8, parcialmente irrompido. Iniciou uso de diclofenaco sódico, por conta própria. $\mathrm{Na}$ sequência, evoluiu para aumento de grande volume em região cervical, associado à febre diária de $38^{\circ} \mathrm{C}$ e disfagia progressiva, que o levou a procurar assistência médica em Unidade de Pronto Atendimento. Na ocasião, foi introduzida antibioticoterapia, com administração endovenosa de clindamicina $600 \mathrm{mg}$, de 6 em 6 horas, e cefatriaxona sódica $1 \mathrm{~g}$, de 12 em 12 horas. Foi encaminhado ao Serviço do Hospital Universitário Professor Edgard Santos para investigação diagnóstica e tratamento.

Ao exame físico extraoral, foi observado edema na região submandibular direita, submentoniana, sublingual e tórax com hiperemia, linfonodo submandibular palpável à esquerda de aproximadamente $2 \mathrm{~cm}$, fibroelástico, móvel e doloroso à palpação. À esquerda, apresentava tumoração em região cervical no trígono mandibular e carotídeo doloroso à palpação, endurecido, de aproximadamente $4 \mathrm{~cm}$ (Figura 1).

Figura 1 - Fotografia extrabucal mostrando celulite cervical à esquerda

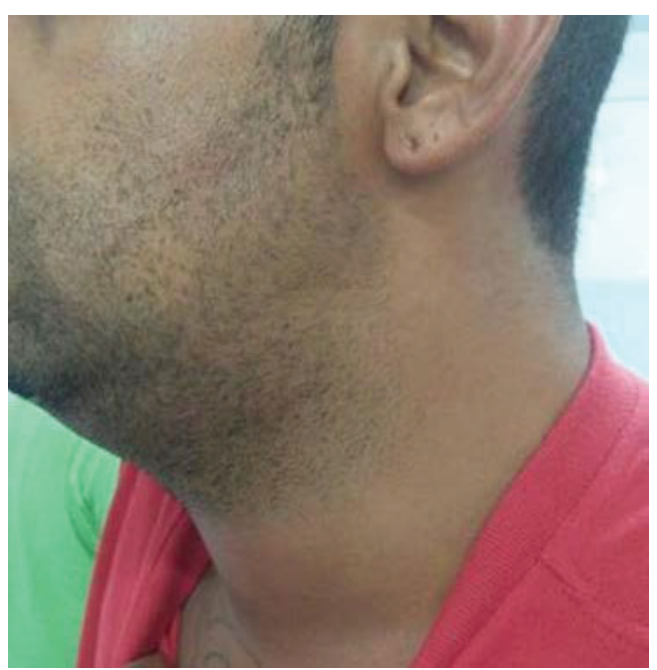

Fonte: Os autores.

Ao exame clínico intraoral, observou-se sialorreia, unidades 38 e 48 semi-irrompidas, com capuz gengival e gengiva da região retromolar inflamada sem supuração. Não foram observadas unidades cariadas ou presença de restos radiculares. Realizaram-se tomografias computadorizadas (TC) de pescoço e de tórax. Na TC, notaram-se extensas coleções hipodensas cervicais multiloculadas, atingindo os espaços sublingual, submandibular, mucoso-faríngeo, parafaríngeo e mesdiastino (Figura 2).

Figura 2 - Mostra as imagens (a),(b),(c), cortes axial, coronale sagital, respectivamente, de TC com janela para tecidos moles, exibindo coleções hipodensas cervicais multiloculadas em toda região faríngea que atingem o mediastino. 

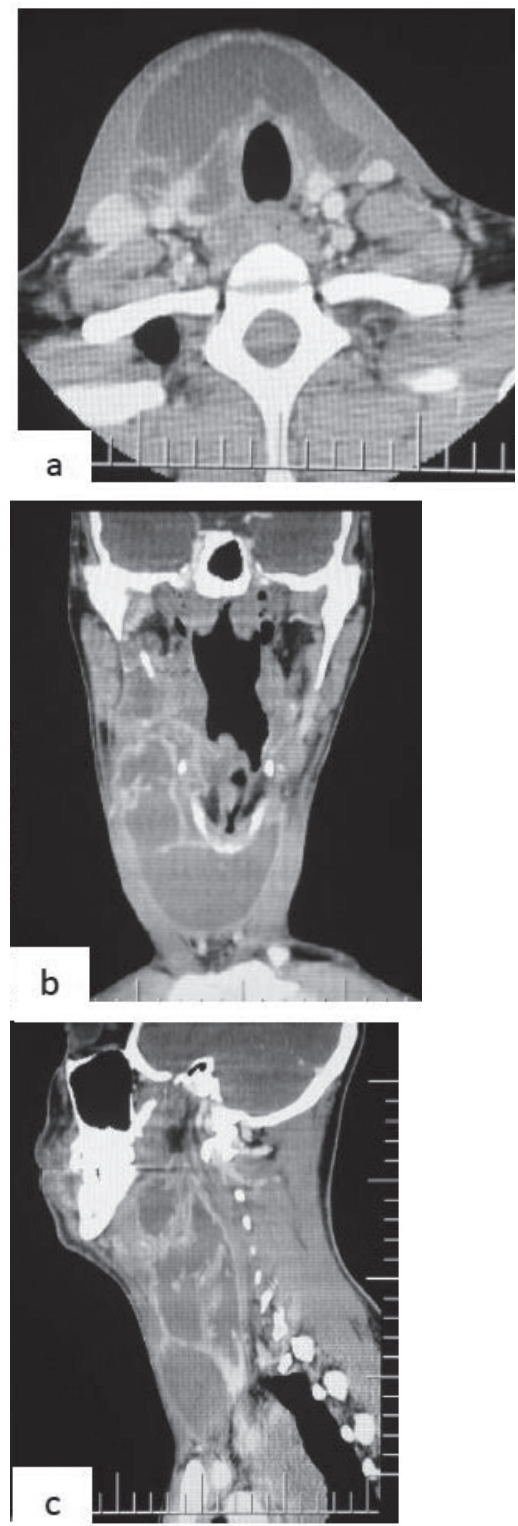

Fonte: Dados da pesquisa.

Os exames laboratoriais admissionais exibem leucocitose sem desvio à esquerda, como mostra a Tabela 1.

Tabela 1 - Exames laboratoriais iniciais da admissão.

\begin{tabular}{l|l}
\hline Hemoglobina & $12,1 \mathrm{~g} / \mathrm{dL}$ \\
\hline Hematócrito & $34,6 \%$ \\
\hline Contagem global de leucócitos & $14580 \mathrm{Leuc} / \mathrm{mm}^{3}$ \\
\hline Bastonetes & $145,0 \mathrm{Leuc} / \mathrm{mm}^{3}$ \\
\hline Segmentados & $12101,4 \mathrm{Leuc} / \mathrm{mm}^{3}$ \\
\hline Linfócitos & $1895,4 \mathrm{Leuc} / \mathrm{mm}^{3}$ \\
\hline Monócitos & $1226,55 \mathrm{Leuc} / \mathrm{mm}^{3}$ \\
\hline Contagem de plaquetas & $503.000 / \mathrm{mm}^{3}$ \\
\hline Proteína C reativa & $67,7 \mathrm{mg} / \mathrm{L}$ \\
\hline Sódio & $135 \mathrm{mEq} / \mathrm{l}$ \\
\hline Potássio & $3,6 \mathrm{mEq} / \mathrm{l}$ \\
\hline Anti HIV I/II & Não reagente \\
\hline
\end{tabular}

Fonte: Dados da pesquisa.

O diagnóstico foi de mediastinite descendente necrosante, associada à angina de Ludwig, secundária à pericoronarite. Realizaram-se, por via endovenosa, analgesia com dipirona sódica $1 \mathrm{~g}$, de 6 em 6 horas, e antibioticoterapia com clindamicina $600 \mathrm{mg}$, também de 6 em 6 horas, associada a ceftriaxona sódica $1 \mathrm{~g}$, de 12 em 12 horas.

Durante o período de internamento, o paciente se manteve estável, com episódios febris esporádicos. Foi realizada drenagem guiada por USG de abscesso cervical, extraindo-se $80 \mathrm{~mL}$ de secreção purulenta, com melhora importante do edema e da hiperemia em região cervical. No $12^{\circ}$ dia de internamento hospitalar, o paciente foi submetido, sob anestesia geral, à cervicotomia, pela equipe de cirurgia torácica, sem intercorrências, mantendo dreno em região cervical anterior, por seis dias, com débito decrescente variando de $75 \mathrm{~mL}$ a $20 \mathrm{~mL} /$ dia.

Após 19 dias de internamento, o paciente recebeu alta hospitalar em bom estado geral, clínica e hemodinamicamente estável, afebril, negando disfagia, dispneia, disfonia e aceitando bem a dieta via oral; submeteu-se ainda à exodontia do dente $3.8 \mathrm{e}$ a acompanhamento ambulatorial.

\section{DISCUSSÃO}

A mediastinite descendente necrosante ocorre normalmente após infecções orofaríngeas e cervicofaciais. $O$ caso apresentado tem etiologia de infecção odontogênica. A MDN que se desenvolve após infecção odontogênica é rara e apresenta alta taxa de mortalidade, chegando a aproximadamente 40\%. (SAID et al., 2014; SARNA et al., 2012). Os achados clínicos frequentemente encontrados são edema e eritema, de início na região superior do pescoço, comumente no soalho da boca, além de dor, febre, disfagia, odinofagia, além de dispneia, estridor e cianose que apontam para um quadro de obstrução de vias aéreas (WOLFE; DAVIS; PARKS, 2011). Alguns desses achados estão de acordo com os observados no caso clínico em questão, no qual o paciente apresentou dor, febre, disfagia e edema na região cervical.

Como exame complementar, foi realizada tomografia computadorizada, sendo importante no auxílio diagnóstico e no plano de tratamento da MDN, pois indica a extensão da lesão, o nível de comprometimento das vias aéreas e permite ao cirurgião a visualização da região cervical e torácica, o que possibilita observar a melhor localização da intervenção cirúrgica, indicada quando há ponto de flutuação ou presença de secreção purulenta, quando o cirurgião realizará o debridamento e a drenagem dos espaços infeccionados (TUCUNDUVA et al., 2013).

$\mathrm{Na}$ antibioticoterapia inicial, referente ao caso clínico em questão, foram administradas em associação as drogas clindamicina e cefatriaxona sódica. É comum a associação antibiótica, em casos de infecções graves; ambas as drogas têm ampla espectro de ação. A clindamicina é comumente 
empregada em caso de paciente alérgico à penicilina e age contra organismos gram-positivos, incluindo anaeróbios e beta-lactamases resistentes. No tratamento da mediastinite odontogênica, a terapia antibacteriana tem sido empregada como uma conduta eficaz. A antibioticoterapia deve ser administrada em longo prazo e os antibióticos empregados devem ser selecionados dentre os grupos que também podem afetar microorganismos resistentes (OPITZ et al., 2015).

A cefatriaxona sódica é uma cefalosporina de $3^{\circ}$ geração, tem ação antibiótica contra microrganismos anaeróbios. Conto et al. (2011) relatam o uso da ceftriaxona sódica em associação com a clindamicina e a gentamicina, na terapia de casos semelhantes.

O paciente ficou internado em ambiente hospitalar durante 19 dias. O tempo de internamento está em acordo com estudos similares, variando de 15 a 90 dias, a depender de fatores como idade e histórico de comorbidades associadas (FERNANDES et al., 2017).

As complicações do tipo mediastinite podem ser letais, culminando consequentemente em risco de morte, deste modo as infecções odontogênicas devem ser monitoradas com cautela; o emprego de antibióticos eficazes e de amplo espectro devem fazer parte da conduta terapêutica. Todavia, se a mediastinite evoluir para uma complicação, deve ser empregada a antibioticoterapia em doses altas e em longo prazo, a drenagem cirúrgica deve ser aplicada no período inicial e o curso da infecção deve ser monitorado de perto após a regressão dos sintomas (SOYLU et al., 2019). No caso clínico em questão, o paciente foi tratado com antibióticos de amplo espectro, realiza-se drenagem guiada por ultrassonografia de abscesso cervical e cervicotomia; e ele permaneceu internado até a resolução do quadro. Após a alta médica, foi encaminhado para a realização de exodontia e permaneceu em acompanhamento ambulatorial.

O prognóstico do caso apresentado foi favorável; isso se deve ao fato da intervenção ter sido realizada em intervalo de tempo suficiente para a resolução satisfatória da infecção.

\section{CONCLUSÃO}

A mediastinite descendente necrosante é uma patologia rara e potencialmente letal, levando frequentemente o paciente a óbito, se não tratada adequadamente. 0 tratamento da MDN é baseado em três pontos principais: diagnóstico precoce, antibioticoterapia de amplo espectro e abordagem cirúrgica. O uso da tomografia computadorizada é imprescindível no auxílio diagnóstico e no planejamento cirúrgico. O cirurgião-dentista deve estar consciente da possibilidade de disseminação pelos espaços faciais e mediastinais dos processos infecciosos de origem odontogênica, bem como estar apto a tratar corretamente os casos, antes que estes acarretem grande risco à vida dos pacientes.

\section{REFERÊNCIAS}

AHMAD, N. et al. The financial burden of hospitalization associated with odontogenic infections. J. oral maxillofac. surg., Philadelphia, v. 71, n. 4, p. 656-658, 2013.

AZENHA, M. R. et al. Celulite facial de origem odontogênica: apresentação de 5 casos. Rev. cir. traumatol. buco-maxilo-fac., Camaragibe, v. 12, n. 3, p. 41-48, 2012.

CHRISTENSEN, B. et al. The cause of cost in the management of odontogenic infections 1: a demographic survey and multivariate analysis. J. oral maxillofac. surg., Philadelphia v. 71, n. 12, p. 2058-2067, 2013.

CONTO, F. et al. Mediastinite de Origem Odontogênica. Rev. cir. traumatol. buco-maxilo-fac., Camaragibe, v.11, n. 2 p. 27, 2011.

EISLER, L. et al. Morbidity and cost of odontogenic infections. Otolaryngol. head neck surg., Rochester, v. 149, n. 1, p. 84-88, 2013.

FERNANDES, B. R. et al. Mediastinite pós infecção odontogênica: relato de caso. Revista Científica Multidisciplinar das Faculdades São José, Rio de Janeiro, v.10, n. 2, 2017.

JUNDT, J. S. et al. Characteristics and cost impact of severe odontogenic infections. Oral surg. oral med. oral pathol. oral radiol., St. Louis, v. 114, n. 5, 2012.

LITTLE, J. W. et al. Dental management of the medically compromised patient. 9. ed. [S.I.]: Elsevier, 2017.

PEREIRA, S. et al. Infecções cervicais - casuística dum serviço de ORL. Revista Portuguesa de Otorrinolaringologia e Cirurgia Cérvico-facial, [s.l], v. 49, n. 3, 2011.

RASTENIENĖ, R. et al. Determinants of length of hospitalization due to acute odontogenic maxillofacial infections: a 2009-2013 retrospective analysis. Rev. Med. Princ. Pactrice, [s.I], v. 24, n. 2, p.129-135, 2015.

SAID, S. et al. Descending necrotizing mediastinitis of odontogenic origin in a young male patient: case report and discussion. Am. J. Med. Case Rep., [s;1], v. 2, n. 2, p. 44-47, 2014.

SARNA, T. et al. Cervical necrotizing fasciitis with descending mediatinitis : literature review and case report. J. Oral Maxillofac. Surg., Philadelphia, v. 70, p. 1342-1350, jun. 2012.

SOYLU, E. et al. Mediastinite como complicação da infecção odontogênica: relato de caso. Niger J. Clin. Pract., Nigeria, v. 22, n.6, p. 869-871, 2019.

SUEHARA, A. B. et al. Infecções cervicais profundas: análise de 80 casos. Braz. J. Otorhinolaryngol., São Paulo, v. 74, n. 2, p. 253-259, 2008.

TUCUNDUVA. M. J. A. P. S. et al. Estudo imaginológico de um caso de angina de Ludwing. Rev. Odontol. Univ., São Paulo, v. 25, n. 2, p. 164169, 2013.

OPITZ, D. et al. Incidência e manejo de infecções odontogênicas graves - uma análise retrospectiva de 2004 a 2011. J. CranioMaxillofac. Surg., New York, v. 43, n. 2, p. 285-289, 2015.

WOLFE, M. M.; DAVIS, J.W. PARKS, S.N. Is surgical airway necessary for airway management in deep neck infections and ludwig angina? J. crit. care, Philadelphia, v. 26, p., n. 1, p. 1-14, 2011.

Submetido em: 07/11/2019

Aceito em: 30/11/2019 